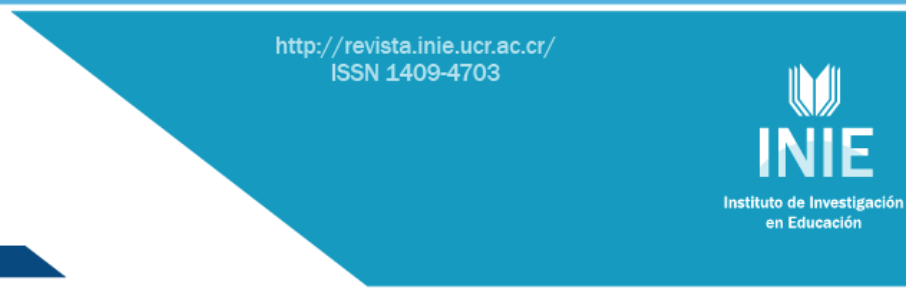

\title{
ALTERNATIVA PEDAGÓGICA PARA LA FORMACIÓN DE GESTORES AXIOLÓGICOS CULTURALES DESDE LA EXTENSIÓN UNIVERSITARIA
}

PEDAGOGICS ALTERNANT FOR THE FORMATION OF AXIOLOGICAL MANAGERS FROM THE UNIVERSITY EXTENSION

Volumen 16, Número 3
Setiembre-Diciembre

pp. 1-19

Dionisio Vitalio Ponce Ruiz Alba Rosa Pupo Kairuz Indira Ferrer González

Dolores Magaly Cedeño Troya

Calixto Guerra González

Revista indizada en REDALYC, $\underline{\text { SCIELO }}$

Revista distribuida en las bases de datos:

LATINDEX, DOAJ, E-REVIST@S, IRESIE, CLASE, DIALNET, SHERPA/ROMEO, QUALIS,

Revista registrada en los directorios:

ULRICH'S, REDIE, RINACE, OEI, MAESTROTECA, PREAL, $\underline{\text { CLACSO }}$ 


\title{
ALTERNATIVA PEDAGÓGICA PARA LA FORMACIÓN DE GESTORES AXIOLÓGICOS CULTURALES DESDE LA EXTENSIÓN UNIVERSITARIA PEDAGOGICS ALTERNANT FOR THE FORMATION OF AXIOLOGICAL MANAGERS FROM THE UNIVERSITY EXTENSION
}

\author{
Dionisio Vitalio Ponce Ruiz' \\ Alba Rosa Pupo Kairuz² \\ Indira Ferrer González ${ }^{3}$ \\ Dolores Magaly Cedeño Troya ${ }^{4}$ \\ Calixto Guerra González ${ }^{5}$
}

\begin{abstract}
Resumen: El presente artículo muestra una estrategia pedagógica para la formación de los gestores culturales con la asunción de nuevas posturas epistemológicas y praxiológicas requeridas por la dinámica formativa de líderes extensionistas universitarios competentes, comprometidos y trascendentes en su actuar a nivel local, construyendo un espacio de redefinición conceptual y metodológica, que se erige sobre el método de la transposición sociocultural. Esta propuesta es el resultado de diversos estudios sobre la formación de profesionales, desarrollados desde la lógica dialéctica, y a partir de un diseño investigativo que ha tenido en cuenta teorías como la complejidad, la teoría general de sistemas, los fundamentos de la pedagogía y la didáctica asociados al desarrollo de la cultura profesional en estudiantes universitarios. Se presentan los resultados de la implementación de esta estrategia en Cuba durante el período 2009-2013 y en Ecuador 2013-2014, para la formación integral de los profesionales como gestores axiológicos culturales, en la que se logran niveles académicos cualificados en ascenso con transferencia en el empoderamiento de estos profesionales, dando fe de la pertinencia social y relevancia como líderes en los barrios e instituciones. Sobre la metodología empleada en el desarrollo de la investigación, se utiliza el paradigma mixto, al ser una investigación cuanti-cualitativa y, de acuerdo con el fin que se persigue, es una investigación de transformación de avanzada, situándose en el tipo propositiva, a partir de establecer una propuesta explicativa causal que deviene en una modelación transformadora del problema científico abordado y que permite instrumentar una nueva práctica pedagógica didáctica en la formación de los gestores culturales.
\end{abstract}

Palabras clave: GESTIÓN CULTURAL, CUBA, ECUADOR, EDUCACIÓN SUPERIOR, PROFESIONALES

\begin{abstract}
This article presents a pedagogical strategy for the training of cultural managers with the assumption of new epistemological and praxiological positions required by the formative dynamics of competent university extension leaders, committed and transcendent in their act locally, building a space of conceptual redefinition and methodological, which stands on the method of sociocultural transposition. This proposal is the result of various studies on the training of professionals, developed from dialectical logic, and from a research design that has taken into account theories like complexity, general systems theory, fundamentals of pedagogy and didactics associated with the development of professional culture among university students. The results of the implementation of this strategy in Cuba are presented for the period 2009-2013 and 2013-2014 Ecuador, for the integral formation of professionals and cultural managers, where academic standards are achieved with skilled rising transfer empowerment of these professionals, attesting to the social relevance and significance as leaders in neighborhoods and institutions. On the methodology used in the development of research, the mixed paradigm, being a quantitative and qualitative research and, according to the aim pursued is used, is a research transformation advanced, reaching the propositional type, from to establish a causal explanatory proposal that becomes a transforming scientific problem addressed modeling and allows implementing a new teaching pedagogical practice in the training of cultural managers.
\end{abstract}

Key words: MANAGEMENT CULTURAL, CUBA, ECUADOR, HIGHER EDUCATION, PROFESSIONAL

\footnotetext{
1 Profesor investigador de la Universidad Regional Autónoma de los Andes, Ecuador.

Dirección electrónica: manzanillo1962@gmail.com

2 Profesora investigadora de la Universidad Regional Autónoma de los Andes, Ecuador.

Dirección electrónica: apupokairuz@gmail.com

${ }^{3}$ Profesora de la Universidad de Granma, Cuba. Dirección electrónica: iferrerg@udg.co.cu

${ }^{4}$ Profesora investigadora de la Universidad Regional Autónoma de los Andes, Ecuador. Dirección electrónica:magaly cedeno@hotmail.com
}

${ }_{5}^{5}$ Profesor investigador de la Universidad de Granma, Cuba. Dirección electrónica: cguerrag@udg.co.cu

Artículo recibido: 20 de abril, 2015 


\section{Introducción}

La necesidad del desarrollo sociocultural en contextos diversos, en medio de las actuales dinámicas posmodernas y globalizantes, ha generado una serie de visiones epistemológicas y de prácticas metodológicas alrededor de esta temática. Es acuciante el interés de desarrollar procesos formativos que permitan, desde las dinámicas extensionistas de las universidades, una mayor socialización del saber y el desarrollo de profesionales competentes en el campo de la gestión cultural, capaces de ejercer un liderazgo activo y transformador que logre la tan ansiada fusión entre cultura universal y cultura local sin marcas hegemónicas.

Este documento es el resultado de una investigación que trabaja las prácticas formativas de gestores socioculturales. Pretende contribuir desde presupuestos epistemológicos y metodológicos a formar profesionales que asuman el desarrollo de los procesos de la gestión sociocultural en las comunidades, ejerciendo un verdadero papel de líderes o guías socioculturales.

Es una experiencia que se ha consolidado a partir de la interacción con los grupos de estudiantes de licenciatura en Estudios Socioculturales de la Sede Universitaria en Manzanillo, Cuba y Ecuador; así como durante los procesos de formación complementaria de sonidistas, productores y otros directivos de espectáculos musicales en los cuales este equipo de investigadores ha participado. Dicha experiencia formativa se ha extendido al territorio ecuatoriano, donde durante los últimos meses se ha estado desarrollando un proceso formativo de gestores universitarios asociados a la investigación y la vinculación cultural.

El proceso investigativo desarrollado permitió fundamentar epistemológicamente un modelo que revela las relaciones y nexos que se dan en el proceso de formación de los gestores socioculturales. A continuación se hace una valoración de cómo se llegó a este modelo y sus particularidades.

\section{Modelo de la dinámica formativa en gestión sociocultural en los profesionales como gestores socioculturales}

\subsection{Referentes epistemológicos}

Para la elaboración del modelo de la dinámica formativa en gestión sociocultural desde lo teórico metodológico se asume como alternativa científica la teoría holística- 
configuracional (Fuentes González, 2009), pues permite revelar el comportamiento del proceso de formación de los profesionales en este campo.

Desde lo pedagógico didáctico, el modelo se fundamenta en la didáctica de la educación superior (Fuentes González, 2009). Dicha teoría permite comprender los procesos de comprensión, explicación, interpretación del proceso de la dinámica formativa en gestión sociocultural, desde la pedagogía y la didáctica de la educación superior. Se considera además la plataforma pedagógica de la Educación Popular (Freire, 1998) dada la dinámica de la formación en contextos culturales y el carácter dialógico cultural de esta, al propiciar en los sujetos la integración reflexiva de los imaginarios socioculturales.

Desde lo antropológico, se consideran importantes los presupuestos con respecto a la transculturación (Ortiz Fernández, 1963), la hibridación cultural (García Canclini, 2005) y la multiculturalidad. Desde lo sociológico, son fundamentales, además, los presupuestos de los cuales se hacen énfasis en la teoría de las representaciones sociales y los imaginarios socioculturales (Moscovici, 1961, Baczko, 1967, Ausubel, Novak y Hanesian, 1999, Maffesoli, 2004 y Franzone, 2007).

Al abordar la construcción social de la realidad, se constata que esta es el resultado de los procesos de intercambios, relaciones y transformaciones que se dan a nivel del pensamiento y el actuar de los sujetos. En este sentido, es manifiesta la importancia que los imaginarios socioculturales tienen en las dinámicas de desarrollo de los grupos humanos en los contextos culturales. Por ello, si, de manera intencional, se trabajan estos procesos, desde la pedagogía y la didáctica de la educación superior, con el interés de formar a los gestores socioculturales, entonces se precisa plantear el concepto de la transposición sociocultural, el cual asume a "la cultura como ente dinamizador de la formación de profesionales en contextos socioculturales diversos" (Ponce Ruiz, 2011, p. 48).

La transposición sociocultural es un constructo que se elabora desde consideraciones pedagógicas relativas a la transculturación y la hibridación cultural, como procesos reconocidos por las Ciencias Sociales. Para el sostén teórico, se ha penetrado en la esencialidad de estos procesos de intercambio cultural, lo que permite plantear que la transposición sociocultural es un complejo proceso de relaciones y transformaciones de los imaginarios socioculturales que favorece la formación de los sujetos a partir de su interrelación en los contextos culturales (Ponce Ruiz, 2011).

De modo que en una dinámica de reconocimiento, apropiación, intervención y validación sociocultural que puede connotar los comportamientos humanos, las prácticas 
culturales y las cosmovisiones, por ello, la transposición sociocultural es un proceso que se da a partir de la relación vivencial entre los sujetos y el contexto cultural en una dinámica de reconocimiento, apropiación, intervención y validación de estadios socioculturales. Se erige por tanto, según Ponce Ruiz (2011), en un "proceso de desarrollo humano que adquiere en el sujeto trascendencia al reconocer las relaciones entre el socio y autodesarrollo como unidad dialéctica, dinamizadora de la esencialidad espiritual y humana".

En esta definición, es significativo el rol que juegan los imaginarios socioculturales por la lógica de apropiación y reconstrucción de las cogniciones y los objetos culturales que funcionan tanto a nivel individual como colectivo, bajo la influencia de la relación y transformación de dichos imaginarios. Esto constituye una posibilidad para las Ciencias Pedagógicas, al abrir un área nueva de explicaciones para la dinámica de significatividad y sentido que se dan en la relación sujeto-contexto social en los procesos de contextualización.

Al tener en cuenta el planteamiento de la transposición sociocultural como soporte pedagógico, se precisa entonces dilucidar el discurrir del modelo de la dinámica formativa de la gestión sociocultural. Este tiene en consideración que el proceso de formación de los licenciados en Estudios Socioculturales como gestores ocurre en un espacio social que hemos llamado espacio socio formativo, el cual está condicionado por la relación academiacontexto cultural, que se singularizan en los ámbitos laborales de estos profesionales, así como "aspectos de carácter ético espiritual que modelan los comportamientos de los seres humanos y que actúan como condicionantes del desarrollo individual y social" (Moya Padilla, 2009, p. 55).

Este proceso formativo sociocultural se constituye en un asunto social que se desarrolla a través de la construcción de significados y sentidos entre los sujetos implicados, en el tiempo y el espacio, siendo esta una construcción dialéctica y coherente orientada hacia la consecución de la condición humana, la cual está revelando la trascendencia del sentido de vida que asumen estos sujetos. Por tanto, es el proceso social intencional formativo en que los sujetos, en el desarrollo de la actividad humana y con un carácter subjetivo-objetivo, desarrollan su cultura y educación, como aspecto esencial de su formación profesional.

Dicho proceso tiene una doble estructuración cuando se modela, pues se constituye a partir de la interrelación de dos planos holísticamente relacionados; el plano de la formación educativa socio espiritual y el plano de la formación cultural profesional, en el cual se 
expresan las dinámicas de la formación de los gestores socioculturales desde lo relacional entre la cultura académica y la cultura de los contextos (Ponce Ruiz, 2011, p. 47).

Se consigna que esta doble relación está revelando la presencia, en el modelo de la dinámica formativa en gestión sociocultural, de elementos que influyen en el proceso formativo de la espiritualidad de los sujetos, a partir de relaciones dialécticas que se establecen entre aspectos como la connotación, la denotación socio profesional, la actuación sociocultural y la actuación socioprofesional, los cuales condicionan y propician el desarrollo socio cultural profesional de estos gestores (Ponce Ruiz, 2011, p. 47).

El modelo permite asegurar la comprensión, explicación e interpretación de la realidad educativa y su vínculo con los contextos culturales, desde un acercamiento a los procesos formativos de los licenciados en Estudios Socioculturales como gestores, a partir de asumir dinámicas que condicionan el reconocimiento de la cultura universal y la cultura de los contextos. Es importante agregar que este modelo está construido para atender procesos formativos en el marco de la semipresencialidad, pero dados sus ascensos epistemológicos y metodológicos, se hace susceptible de una generalización en diversos ámbitos de la formación de profesionales. Dicho modelo tiene como pilar, en su base epistemológica, a la dialéctica-materialista, la que permite la revelación del movimiento del objeto y de todo el sistema de relaciones dialécticas que en él se establece a partir de la consideración del imaginario sociocultural como un referente social propiciador de la mediación cultural. De igual manera, permite develar las configuraciones y dimensiones que emergen de dicho movimiento.

Para su construcción. este modelo parte de tres ideas básicas:

- La existencia de un proceso de formación sociocultural que tiene sus fundamentos en la relación universidad-contexto cultural, como estructuración espacial y temporal de la lógica formativa de la profesión del licenciado en Estudios Socioculturales.

- La búsqueda epistemológica de la solución a la contradicción entre la apropiación de la diversidad sociocultural en el actuar de estos licenciados y la necesidad de una dinámica formativa transdisciplinar de la gestión sociocultural que enriquezca sus modos de actuación profesional. Ello condiciona el desarrollo de la capacidad sociocultural transformadora como matriz profesionalizante de estos licenciados, en la cual se produce la integración reflexiva dialógica sociocultural, que se erige en cualidad proyectiva que sintetiza la relación entre expectativas profesionales y trascendencia sociocultural. 
- La relación entre expectativas profesionales y trascendencia social del gestor, desde lo individual y lo social, como expresión de las potencialidades formativas del diálogo cultural que se da en los sujetos en formación.

Todo lo cual queda graficado en la representación del modelo desarrollado que a continuación se presenta (Figura 1).

Figura 1

Representación del modelo de formación de la gestión sociocultural

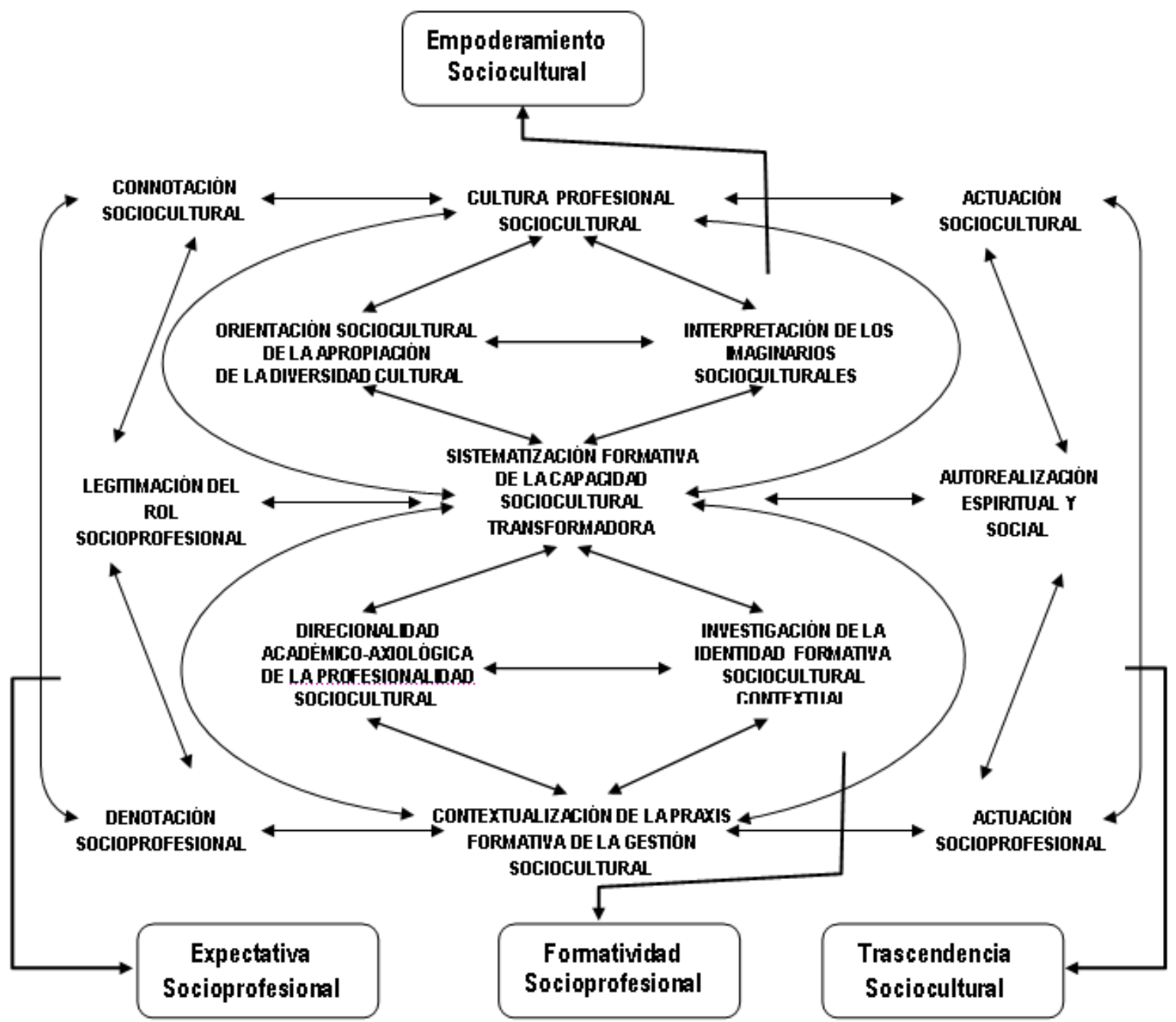

Fuente: Ponce Ruiz, 2011. 


\subsection{Argumentación científica metodológica del modelo de la dinámica formativa en gestión sociocultural}

Al hacer un análisis que sitúe la trascendencia que el modelo puede tener para la educación superior cubana, es preciso consignar que las relaciones holísticas entre las dimensiones reveladas en el proceso investigativo, que se sintetizan en el constructo sistematización formativa de la capacidad sociocultural transformadora, dan cuenta de las expectativas que el gestor encuentra en su espiral socio formativa desde la validación de sus necesidades y aspiraciones socio profesionales.

Se constituye en el punto de partida que en cualquier instante de su formación profesional tiene esta persona. Es decir que en el orden del desarrollo del proceso de formación durante la carrera en cualquier estadio en el cual se encuentre el estudiante en él se van a dar necesidades de legitimación de su rol profesional. Por ello, al considerar la entrada a un curso, disciplina o asignatura, "siempre estarán presentes determinados objetivos que el proceso docente va a requerir y que van a constituir referentes desde lo académico que permitirán al estudiante tener una percepción de sus necesidades de formación". (Ponce Ruiz, 2011, p. 64)

Lo anterior confiere a las expectativas socio-profesionales el carácter continuo de dirección de los objetivos que el estudiante se trazara para la obtención de logros en su proceso formativo, lo cual representa una direccionalidad en el desarrollo profesional del sujeto. Al tanto que la trascendencia sociocultural se erige en intencionalidad dialéctica, da cuenta de la trascendencia humana ante la sociedad y la vida. Se da a partir de un pensamiento holístico, creativo y critico a través de una comunicación significativa y creativa lograda en una interrelación social afectiva que propicie el desarrollo de la autoestima, autoconocimiento y socio progresión espiritual. Posibilitando una sólida conciencia moral y ética, desde una sensibilidad estética a partir de una conciencia holística de lo ambiental, lo histórico y lo humano que potencien las habilidades para el trabajo y la vida con un sentido de trascendencia que dinamiza la libertad y la elegibilidad responsable.

Esta dimensión permite que el proceso de formación examine nuevas búsquedas epistemológicas y metodológicas en el sujeto que se forma, constituyendo una dinámica de socio-progresión cultural en una espiral infinita del desarrollo de las potencialidades humanas de estos estudiantes. Es así como la relación entre las expectativas socioprofesionales y la trascendencia sociocultural se constituye en eje integrador y dinamizador de una ética formativa que va a condicionar el desarrollo de valores en el estudiante que se 
forma como profesional de la gestión sociocultural.

En otro plano de modelación del proceso e integrado a la percepción holística de este, se da el carácter puramente profesional en la sistematización epistemológica y metodológica, donde la dimensión empoderamiento sociocultural condiciona una nueva manera de entender el accionar de los licenciados en Estudios Socioculturales y expresa los procesos que se suscitan con el interés de desarrollar en el gestor, capacidades, competencia y habilidades que le permitan intervenir con poder de transformación en los procesos socioculturales. Esta dimensión resignifica el accionar consciente y transformador en los sujetos y va potenciar sus maneras de intervenir en los procesos de desarrollo sociocomunitario como verdaderos agentes de cambio.

Al considerar la dimensión formativa sociocultural, la cual expresa los procesos formativos que se dan en la universidad en función de la formación de estos gestores socioculturales, es importante significar cómo configura la formación en el sujeto de habilidades profesionales que dan cuenta de la sistematización epistemológica y metodológica de la profesión.

En resumen, la riqueza de este modelo se expresa en la manera en que la sistematización formativa de la capacidad sociocultural transformadora, la cual expresa las potencialidades del ser humano para participar de forma proactiva en los cambios culturales que ocurren en la sociedad, es integradora de las dimensiones antes expuestas en una lógica de espiral socio formativa que privilegia el diálogo cultural, en ascensos propios del reconocimiento de los significados y los sentidos culturales para proponer una dinámica formativa que mediatiza las discrepancias entre educación tradicional racionalista y educación contextual desde la tradición cultural de los contextos.

Se ha de resaltar que la capacidad sociocultural transformadora da cuenta entonces de las potencialidades holísticas creadas en el gestor sociocultural para observar, comprender, explicar e interpretar los procesos socioculturales desde una mirada trascendente a su profesión que legitima su papel en la sociedad. (Fuentes González, 2009, p. 71)

Siendo, por tanto, la sistematización de la capacidad sociocultural transformadora el constructo esencial de este modelo al interpenetrar en otras configuraciones, como necesaria, la cualidad resultante de la formación progresiva del gestor, esta sistematización se va dando en un proceso vivencial, en el cual, el gestor se empodera de la cultura y actúa consecuentemente con ella. Permitiéndole a través de procesos cognitivos intencionados la 
autoregulación de su aprendizaje y participar activamente en la dinámica interventora de la profesión.

Al hacer un análisis del constructo teórico esencial del modelo y sus relaciones con la configuración formación de la cultura profesional sociocultural y la de contextualización de la praxis formativa de la gestión sociocultural, se concluye que este parte de la práctica formativa en la cual el estudiante es capaz de sistematizar su capacidad sociocultural transformadora, lo cual propicia la concreción de un ciclo de formación de la gestión sociocultural. Siendo muy importante comprender cómo se desarrolla ese ciclo formativo, pues el condiciona la posibilidad de elaborar un método que permita la intervención en los procesos formativos de los estudiantes de la carrera de licenciatura en Estudios Socioculturales.

En esencia, se producen accesos formativos enmarcados en la lógica siguiente: en la contextualización de la praxis formativa de la gestión sociocultural, en busca de apropiarse el estudiante de un determinado contenido cultural, se condiciona un dialogo cultural a nivel de la relación cultura académica y cultura contextual que es resuelto por el individuo desde la integración de la observación, la comprensión y la explicación, lo cual se da a partir de la realización de una integración dialógica que con un carácter reflexivo este realiza y donde se dinamizan saberes previos con las nuevas informaciones culturales susceptibles de ser asimiladas por el sujeto.

Lo anterior condiciona una interpretación de la cultura que conduce a una relación y transformación en el sujeto de su imaginario sociocultural, lo cual significa que se han producido en él, cambios a nivel cognitivo que evidencian una sistematización formativa tanto epistemológica como metodológica que conduce a ascensos en su formación cultural profesional y la cual los empodera para la realización de su cultura profesional en el marco de sus prácticas laborales. Esto es congruente con la dinámica de ascensos en su realización social y espiritual como corroboración de la legitimación de su profesión.

Es significativo que la existencia de esta modelación del proceso formativo de los estudiantes de la licenciatura en Estudios Socioculturales, que tiene en cuenta la dinámica relacional entre la cultura universal y la cultura contextual como esencia dialógica que permite reconocerla en plataforma epistemológica sobre la cual construyo el método de intervención en la práctica formativa de la carrera, se argumenta en el acápite siguiente. 


\subsection{El método de la transposición sociocultural}

Es expresión de las regularidades del modelo de la dinámica formativa de la gestión sociocultural. Parte de las relaciones que se establecen entre sus dimensiones y se encamina a orientar y conducir el proceso de formación socio-profesional y espiritual de los gestores. "Este Método activa el proceso de construcción cultural de las dinámicas de intervención socio-comunitario por parte de los estudiantes". (Ponce Ruiz, 2011, p. 70)

El objetivo del método es, por lo tanto, la edificación de un proceso de autodesarrollo social y espiritual mediado por la cultura instituyente de los imaginarios socioculturales en el desarrollo de conocimientos, valores y actitudes que le permitan al gestor sistematizar su capacidad sociocultural transformadora desde una actuación comprometida, flexible, trascendente y espiritualmente sensible en su rol. Actuación condicionada por la orientación que posibilita en el gestor sociocultural, su integración reflexiva dialógica sociocultural, la cual se expresa como cualidad esencial de estos profesionales en su dialogicidad transpositiva sociocultural.

El método propicia la socio-gestión por parte del gestor de sus conocimientos, conceptos, experiencias, representaciones sociales e imaginarios, en un proceso vivencial de valoraciones relacionadas con la cultura que se da en el dialogo entre academia y contexto, posibilitando el desarrollo de competencias para redireccionar esta información, estableciendo inferencias y deducciones que posibilitan desde proceso de transposición sociocultural los ascensos cognitivos de los individuos. Por lo tanto, la transposición sociocultural se constituye en el principio regulador que sustenta el método desde la funcionalidad lógica, metodológica y gnoseológica que dirige didácticamente la interpretación sociocultural. Permite descubrir los nuevos significados que emergen del contexto cultural y la creación de nuevos sentidos en la medida en que enfrenta desafíos cognitivos. De esta manera, constituye un medio eficaz para la apropiación creativa de los conocimientos, ya que establece una nueva correlación entre la apropiación reproductiva, la productiva y la creativa siendo, estos elementos, base del método propuesto que integra un cúmulo de experiencias y un alto grado de independencia y actividad creadora.

En lo particular, el método de la transposición sociocultural se desdobla en procedimientos que evidencian las relaciones y nexos que se establecen en el proceso de formación de la transposición sociocultural y que promueven un salto cualitativo, de lo viejo a lo nuevo y de lo dogmático a lo flexible-dialéctico. Repercute de manera significativa en la actividad que despliegan los sujetos durante su formación y en su apropiación de la cultura 
contextual. Representa un salto cualitativo en el sistema de relaciones que se establecen en el proceso formativo, por medio del cual se genera el desarrollo del docente y del estudiante, pues hace efectiva la aplicación de la trasposición sociocultural en el proceso de la creación y la co-creación de las representaciones y los imaginarios sociales.

En síntesis, el método es una manera singular de configurar el sentido y el contenido de la enseñanza mediante la creación y la co-creación del contenido cultural en el proceso de transposición sociocultural.

\subsection{Procedimientos del método de la transposición sociocultural}

Los procedimientos contribuyen a la solidez teórico-práctica de la transposición sociocultural y constituyen los momentos esenciales para su concreción.

1. Procedimiento de formación del reconocimiento, apropiación y validación sociocultural.

2. Procedimiento de formación de la dialogización sociocultural.

3. Procedimiento de formación de la corroboración formativa sociocultural.

Estructura del procedimiento de reconocimiento, apropiación y validación sociocultural.

- Reconocimiento sociocultural.

- Apropiación sociocultural.

- Validación sociocultural.

Estructura del procedimiento de formación de la dialogización sociocultural.

- Estructuración de cultura transmisible.

- Selección de estrategias de intervención socio-transformadoras.

- Dinamización de la contextualización sociocultural.

Estructura del procedimiento de la corroboración formativa sociocultural

- Evaluación autoformativa.

- Reprogramación sociointerventora-formativa.

\section{Estrategia pedagógica contextualizada}

La intencionalidad del desarrollo de una estrategia pedagógica que tenga en cuenta el contexto formativo, los preconceptos y los grados de sistematización cultural con que cuentan los involucrados en el proceso, es básicamente el desarrollo de procesos de 
empoderamiento sociocultural que potencien la capacidad dialógica y participativa de los gestores culturales.

\subsection{Estructura funcional}

Se establecen 3 etapas para el desarrollo de la estrategia, ellas permiten dinamizar un ciclo de crecimiento en espiral de los actores involucrados en el proceso formativo empoderante que se ejecuta, la lógica funciona desde la solución de las contradicciones dialécticas que aparecen tanto a nivel del individuo como del grupo formativo. Las etapas son:

\subsubsection{Diseño y planeación proyectiva}

Se procede al diseño y planeación del proceso formativo-empoderante, estableciendo los núcleos teóricos conceptuales básicos, las habilidades y competencias por desarrollar y los modos de participación y relación en la dinámica de dialogización sociocultural formativa que potencie el tramado de relaciones tanto humanas como conceptuales que dan soporte a la estrategia.

Se desarrollan diversos momentos y ciclos de acciones que pueden ser signados como:

- Génesis proyectiva,

- Cohesión proyectiva,

- Co-creación profesoral,

- Estimación nivel de partida,

- Definir grupos colaborativos.

Instituciones participantes

$\checkmark$ Sede Central UDG,

$\checkmark$ FUM Manzanillo,

$\checkmark$ Museo Obrero,

$\checkmark$ Biblioteca Antonio Maceo,

$\checkmark$ Casa de la Trova,

$\checkmark$ Sede Municipal Uneac,

$\checkmark$ Sede AHS,

$\checkmark$ Centro Navarro Luna. 


\subsubsection{Interventora socioformativa}

En esta etapa se dinamizan las acciones directas para el crecimiento de los procesos relacionales a nivel de aula, grupos investigativos e interacción con la comunidad en una constante redefinición de las conceptualizaciones trazadas en la etapa anterior y propiciando la transformación de los niveles de sistematización cultural de los participantes desde la reconstrucción de las teorías, los conceptos, las practicas que singularizan los sociocultural extensionista.

Se propician lógicas y espacios de:

- Interaccionismo áulico,

- Interaccionismo socioinvestigativo,

- Investigación en instituciones,

- Interaccionismo socio productivo,

Sistema docente

$\checkmark$ Conferencias temáticas,

$\checkmark$ Clases orientadoras,

$\checkmark$ Paneles y charlas,

$\checkmark$ Seminarios,

$\checkmark$ Consultas,

$\checkmark$ Tutorías,

$\checkmark$ Sede Municipal Uneac.

\subsubsection{Evaluativa sociocultural proyectiva}

Constituye la etapa evaluativa de la experiencia, en ella se estructuraron los procesos de auto, co y heteroevaluación, involucrando tanto el desarrollo formativo de estudiantes y docentes así como otros actores que aportaron de manera activa al proceso.

Esta etapa es decisiva para la reconfiguración de las acciones futuras y permitió revisar los procesos desarrollados. Se singularizo en:

- Examen culminación de estudio,

- Validación del taller,

- Reconfiguración proyectiva. 
Áreas temáticas

$\checkmark$ Metodología de investigación social,

$\checkmark$ Sociología de la cultura,

$\checkmark$ Cultura cubana,

$\checkmark$ Política cultural,

$\checkmark$ Promoción / Gestión SC.

\subsection{Experiencia socioformativa}

Esta experiencia socioformativa congregó a su amparo a un grupo amplio de docentes de la Facultad Universitaria Municipal de Manzanillo y contó con la colaboración de profesores de la Sede Central de la Universidad de Granma, Cuba, en un período de cuatro cursos.

Se inició como solución perentoria a las deficiencias que expresaban los alumnos al realizar sus exámenes de culminación de la carrera de Estudios Socioculturales. Con la anuencia de la Universidad de Granma y la Sede Municipal de Manzanillo, se desarrollaron una serie de reuniones, diálogos y entrevistas para dar bases proyectivas a la idea de establecer la estrategia. Asumiendo que se contaba con el método de la transposición sociocultural y las experiencias obtenidas en diversos cursos de formación de gestores culturales, por parte de varios docentes involucrados en la carrera, se procedió a convocar a los estudiantes que se encontraban en años terminales, estableciéndose diversas formas de interacción asociadas a las etapas de la estrategia que fueron desarrollándose como estaba establecido.

Las experiencias obtenidas en Cuba han sido generalizadas en talleres formativos desarrollados en la Universidad Uniandes del Ecuador, puesto que en este recinto se expresan necesidades formativas para el desarrollo de las capacidades investigativas en los docentes.

La dinámica de la experiencia responde al desarrollo mejorado de un ciclo de gestión formativa donde cada una de las etapas constituye un tránsito en el proceso de formación y empoderamiento de las funciones que un gestor sociocultural debe potenciar en su interés de ser realmente trascendente durante su actuación profesional, desarrollándose desde la implementación, la teoría sistémica y el método sistémico estructural funcional.

Sobre la metodología empleada en el desarrollo de la investigación, se puede referir que esta se desarrolla en el paradigma mixto, al ser una investigación cuanti-cualitativa y de 
acuerdo con el fin que se persigue, es una investigación de transformación de avanzada, situándose en el tipo propositiva, a partir de establecer una propuesta explicativa causal que deviene en una modelación transformadora del problema científico abordado y que permite instrumentar una nueva práctica pedagógica didáctica en la formación de los gestores culturales. De este modo, se enfoca al desarrollo de una investigación aplicada en el ámbito del pre experimento, que en su momento emplea lógicas de tipo cualitativa al dimensionar la experiencia pedagógica desde la dinámica de la investigación acción participativa.

La población y la muestra coinciden, pues en los cuatro años que se desarrolló la experiencia, la aplicación de los instrumentos siempre estuvo ajustada a menos de 100 personas.

\subsubsection{Instrumentos, materiales consumibles y/o fuentes de datos}

Se emplearon métodos de carácter teórico, empíricos y estadísticos matemáticos. Como se describe en la Tabla 1:

\begin{tabular}{|c|c|c|c|c|}
\hline \multicolumn{5}{|c|}{$\begin{array}{c}\text { Tabla } 1 \\
\text { MÉTODOS CIENTÍFICOS EMPLEADOS EN EL DESARROLLO DE LA INVESTIGACIÓN }\end{array}$} \\
\hline \multirow{2}{*}{$\begin{array}{l}\text { MÉTOD } \\
\text { OS } \\
\text { TEÓRIC } \\
\text { OS }\end{array}$} & \multicolumn{4}{|c|}{$\begin{array}{l}\text { Análisis-síntesis, Inducción-deducción, Histórico lógico, holístico dialéctico, la modelación y el } \\
\text { sistémico estructural funcional }\end{array}$} \\
\hline & FUNCIÓN & $\begin{array}{l}\text { TÉCNICAS y/o } \\
\text { PROCEDIMIENTOS }\end{array}$ & $\begin{array}{l}\text { INSTRUME } \\
\text { NTOS }\end{array}$ & $\begin{array}{l}\text { TAREAS DE } \\
\text { INVESTIGACI } \\
\text { ÓN }\end{array}$ \\
\hline 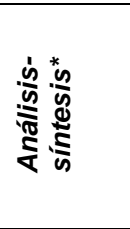 & $\begin{array}{l}\text { La función del método de análisis-síntesis consiste } \\
\text { en establecer las partes que y rasgos que } \\
\text { componen un proceso. Se emplea para la } \\
\text { fundamentación teórica del objeto de } \\
\text { investigación, del campo de acción y en la } \\
\text { modelación de ambos y del instrumento } \\
\text { metodológico. }\end{array}$ & $\begin{array}{ll}\text { Análisis } & \text { de } \\
\text { contenido. } & \text { Fichaje: } \\
\text { desagregado de las } & \text { de } \\
\text { partes y } & \text { síntesis } \\
\text { conceptual } & \end{array}$ & $\begin{array}{l}\text { Fichas } \\
\text { nemotécnica } \\
\mathrm{s} \quad \text { y } \\
\text { bibliográfica } \\
\text { s }\end{array}$ & $\begin{array}{l}\text { Se evidencia a } \\
\text { lo largo de } \\
\text { todo el trabajo } \\
\text { de } \\
\text { investigación. }\end{array}$ \\
\hline 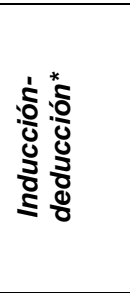 & $\begin{array}{l}\text { Para establecer los razonamientos generales y } \\
\text { particulares del objeto de investigación y el campo } \\
\text { de acción, así como concretar la elaboración } \\
\text { teórica del modelo. Posibilita el movimiento del } \\
\text { pensamiento del todo a las partes y de las partes } \\
\text { al todo en el razonamiento científico para dilucidar } \\
\text { rasgos, especificidades, generalidades y asumir } \\
\text { críticamente posiciones epistemológicas. }\end{array}$ & $\begin{array}{l}\text { Método lógico } \\
\text { deductivo deductivo } \\
\text { Método } \\
\text { directo - inferencia o } \\
\text { conclusión inmediata } \\
\text { Método deductivo } \\
\text { indirecto - inferencia o } \\
\text { conclusión mediata - } \\
\text { formal }\end{array}$ & $\begin{array}{l}\text { Fichas } \\
\text { nemotécnica } \\
\text { s y y } \\
\text { bibliográfica } \\
\text { s, estudios } \\
\text { de hojas de } \\
\text { vida } \\
\text { docente. }\end{array}$ & $\begin{array}{l}\text { Se evidenciará } \\
\text { a lo largo de } \\
\text { todo el trabajo } \\
\text { de } \\
\text { investigación. }\end{array}$ \\
\hline 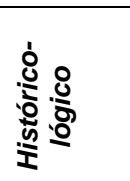 & $\begin{array}{l}\text { Se emplea para determinar la evolución histórica } \\
\text { del objeto y campo de estudio permitiendo } \\
\text { caracterizar de manera tendencial el proceso de } \\
\text { formación de profesionales de la gestión cultural } \\
\text { en Cuba y la Universidad de Granma. }\end{array}$ & $\begin{array}{lr}\text { Análisis } & \text { lógico } \\
\text { histórico, } & \text { fichaje } \\
\text { nemotécnico } & \end{array}$ & $\begin{array}{l}\text { Fichas de } \\
\text { documentos } \\
\text { históricos }\end{array}$ & $\begin{array}{l}\text { En el marco } \\
\text { teórico }\end{array}$ \\
\hline 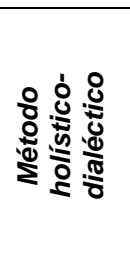 & $\begin{array}{l}\text { Este método permite la observación, comprensión } \\
\text { interpretación y explicación de los fenómenos } \\
\text { estudiados empleándose como plataforma } \\
\text { epistémica para dinamizar los tránsitos cognitivos } \\
\text { dilucidados en la construcción de la propuesta } \\
\text { teórica. Además, permite la estructuración de } \\
\text { procedimientos que den efectividad al modelo } \\
\text { didáctico desarrollado. }\end{array}$ & $\begin{array}{l}\text { Configuraciones del } \\
\text { proceso } \\
\text { Dimensiones del } \\
\text { proceso } \\
\text { Eslabones del proceso }\end{array}$ & $\begin{array}{l}\text { Fichas } \\
\text { nemotécnica } \\
\mathrm{s} \quad \text { y } \\
\text { bibliográfica } \\
\mathrm{s}\end{array}$ & $\begin{array}{lr}\text { Direcciona } & \text { el } \\
\text { proceso } & \text { de } \\
\text { gestión } & \\
\text { investigativa. }\end{array}$ \\
\hline
\end{tabular}




\begin{tabular}{|c|c|c|c|c|}
\hline$\frac{2}{\frac{2}{0}}$ & $\begin{array}{l}\text { Se emplea para desarrollar el modelo de gestión } \\
\text { de la formación del profesional sociocultural. Es } \\
\text { justamente el método mediante el cual se crean } \\
\text { abstracciones con vistas a explicar la realidad. }\end{array}$ & Modelación teórica & $\begin{array}{l}\text { Organizador } \\
\text { es gráficos: } \\
\text { esquemas, } \\
\text { mapas }\end{array}$ & \begin{tabular}{lr}
\multicolumn{2}{l}{ Determinación } \\
del modelo \\
actual \\
planteo del \\
modelo \\
transformador.
\end{tabular} \\
\hline 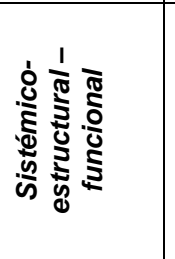 & $\begin{array}{l}\text { Se emplea para caracterizar el sistema } \\
\text { pedagógico didáctico empleado en la actualidad } \\
\text { en la Universidad de Granma para la formación de } \\
\text { los licenciados en estudios socioculturales. }\end{array}$ & $\begin{array}{ll}\text { Determinación } & \text { de } \\
\text { componentes, } & \\
\text { Determinación } & \text { de } \\
\text { estructura, } & \\
\text { Determinación } & \text { de } \\
\text { relaciones, } & \\
\begin{array}{l}\text { Determinación } \\
\text { funciones. }\end{array} & \text { de } \\
\end{array}$ & $\begin{array}{l}\text { Organizador } \\
\text { es gráficos }\end{array}$ & $\begin{array}{l}\text { Para la } \\
\text { estrategia de } \\
\text { intervención } \\
\text { pedagógica } \\
\text { didáctica }\end{array}$ \\
\hline \multirow{2}{*}{ 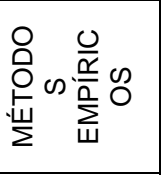 } & \multicolumn{4}{|c|}{ La observación, el experimento pedagógico, la encuesta, la entrevista y el criterio de expertos } \\
\hline & TIPOS Y FUNCIONES & $\begin{array}{l}\text { TÉCNICAS y/o } \\
\text { PROCEDIMIENTOS }\end{array}$ & $\begin{array}{l}\text { INSTRUME } \\
\text { NTOS }\end{array}$ & $\begin{array}{c}\text { TAREA DE } \\
\text { INVESTIGACI } \\
\text { ÓN }\end{array}$ \\
\hline $\begin{array}{l}\text { La } \\
\text { observació } \\
n\end{array}$ & $\begin{array}{l}\text { Se empleará en el desarrollo de la investigación } \\
\text { para tomar datos e información de primera fuente } \\
\text { sobre el comportamiento del objeto de estudio } \\
\text { declarado. }\end{array}$ & \begin{tabular}{|l|} 
Observación \\
sistemática \\
observación \\
participativa
\end{tabular} & $\begin{array}{l}\text { Guía de } \\
\text { observación }\end{array}$ & $\begin{array}{l}\text { Estudio } \\
\text { campo }\end{array}$ \\
\hline \multirow[b]{2}{*}{ 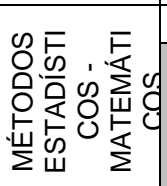 } & \multicolumn{4}{|c|}{ Estadística descriptiva, métodos de graficación matemática } \\
\hline & TIPOS Y FUNCIONES & $\begin{array}{l}\text { TÉCNICAS y/o } \\
\text { PROCEDIMIENTOS }\end{array}$ & $\begin{array}{l}\text { INSTRUME } \\
\text { NTOS }\end{array}$ & $\begin{array}{l}\text { TAREA DE } \\
\text { INVESTIGACI } \\
\text { ÓN }\end{array}$ \\
\hline $\begin{array}{l}\text { Estadísti } \\
\text { ca } \\
\text { descripti } \\
\text { va }\end{array}$ & $\begin{array}{l}\text { Para instrumentar el proceso de diagnóstico del } \\
\text { problema a nivel del contexto en el cual se sitúa } \\
\text { y para evaluar el procesamiento del método de } \\
\text { criterio de expertos empleado. }\end{array}$ & \begin{tabular}{ll|} 
Identificación & de \\
población y muestra
\end{tabular} & Ecuaciones & $\begin{array}{l}\text { Estudio } \\
\text { campo }\end{array}$ \\
\hline \multicolumn{5}{|c|}{ Técnicas de encuesta y entrevista } \\
\hline La encuesta. & $\begin{array}{l}\text { Se realizan a la población seleccionada en el } \\
\text { caso de la encuesta. }\end{array}$ & Estructurada & $\begin{array}{l}\text { Cuestionario } \\
\text { s }\end{array}$ & $\begin{array}{l}\text { Estudio } \\
\text { campo }\end{array}$ \\
\hline $\begin{array}{l}\text { La } \\
\text { entr }\end{array}$ & $\begin{array}{l}\text { La entrevista se orientará a los actores o } \\
\text { informantes claves del proceso. }\end{array}$ & $\begin{array}{l}\text { Estructurada } \\
\text { estructurada }\end{array}$ & $\begin{array}{l}\text { Cuestionario } \\
\mathrm{s}\end{array}$ & $\begin{array}{l}\text { Estudio } \\
\text { campo }\end{array}$ \\
\hline
\end{tabular}

Fuente: Elaborada por los autores.

\subsection{Experiencia en Cuba}

\subsubsection{Resultados académicos}

Tabla 2

\begin{tabular}{|c|c|c|c|c|c|c|c|}
\hline Curso & $\begin{array}{c}\text { A } \\
\text { presentar }\end{array}$ & Presentados & Aprobados & \% aprobados & $\begin{array}{c}\text { Nota } \\
\mathbf{3}\end{array}$ & $\begin{array}{c}\text { Nota } \\
\mathbf{4}\end{array}$ & $\begin{array}{c}\text { Nota } \\
\mathbf{5}\end{array}$ \\
\hline $2009-2010$ & 47 & 40 & 25 & 62,5 & 11 & 8 & 6 \\
\hline $2010-2011$ & 41 & 37 & 14 & 37,87 & 7 & 4 & 3 \\
\hline $2011-2012$ & 82 & 56 & 34 & 60,71 & 18 & 12 & 4 \\
\hline $2012-2013$ & 56 & 33 & 20 & 60,6 & 11 & 4 & 5 \\
\hline Totales & $\mathbf{2 2 6}$ & $\mathbf{1 6 6}$ & $\mathbf{9 3}$ & $\mathbf{5 5 , 4 2}$ & $\mathbf{4 7}$ & $\mathbf{2 8}$ & $\mathbf{1 8}$ \\
\hline
\end{tabular}

Fuente: Elaboración propia de los autores (2013). Resultados comparativo de alumnos presentadosaprobados en Cuba. 
Como se observa, en la Tabla 2, se evidencian resultados positivos en comparación con los procesos formativos empoderantes anteriores y en los cuales los índices de aprobados eran extremadamente bajos. Es importante observar que un índice promedio de 55,42 , para estudiantes que son en su mayoría trabajadores, se convierte en el contexto de la Universidad de Granma en un valor significativo que da cuenta del impacto del proceso desarrollado.

\subsection{Experiencia en Ecuador}

Desde abril de 2014 se viene trabajando el Proyecto GestComplnv, que dinamizado desde la teoría de la transposición sociocultural y su método; este pretende desarrollar grupos investigativos y de intervención cultural en los contextos asociados a la Universidad Uniandes y sus sedes de Santo Domingo de los Tsachilas y Quevedo.

En este marco se desarrolla una contextualización de la estrategia pedagógica que ha cumplido su dos primeras etapas y que evidencia resultados significativos en cuanto a la generación de proyectos de vinculación investigativa extensionista, contándose con más de 10 proyectos activos, que al propio tiempo permiten dimensionar diversos quehaceres locales, como la gestión cultural artística, la gestión gastronómica culinaria y la gestión social inclusiva. Lo anterior ha potenciado la producción científica en este entorno, condicionando 12 artículos enviados a diversas revistas científicas indexadas.

\subsubsection{Aportes científicos alcanzados}

El desarrollo de estos casi cinco años de intervención en los contextos formativos y la dinamización de lógicas dialógicas desde el método han corroborado los aportes que dicha experiencia ha dado signándose:

Aporte teórico: La teoría de la transposición sociocultural sustenta el modelo de la dinámica formativa de la gestión sociocultural, que expresa las relaciones entre la cultura académica y la cultura contextual, en relación con el contenido de los procesos formativos, en los cuales el modelo permite desarrollar en los gestores socioculturales la capacidad sociocultural transformadora en la gestión contextualizada formativa de estos, desde la impronta de los imaginarios socioculturales.

Aportes prácticos: El método de la transposición sociocultural es susceptible de llevarse a la práctica pedagógica por parte de los profesores y estudiantes de la carrera de Estudios Socioculturales, con la intención de empoderar a ambos en el desarrollo de su 
actuación profesional. Se concreta en la estrategia pedagógica para la formación de líderes socioculturales en contextos

Otros aportes: La multimedia Gestión Cultural y el diplomado Gestión Cultural, así como el proyecto de maestría en Gestión Cultural de Avanzada.

La introducción de estos resultados científicos ha evidenciado la valía teóricometodológica del modelo y del método de la transposición sociocultural.

\section{Conclusiones}

La investigación desarrollada arroja los siguientes resultados:

1. Con el modelo de la dinámica formativa de la gestión sociocultural como aporte teórico se enriquece la visión científica de la formación de los gestores socioculturales a partir de la observación, comprensión, explicación e interpretación de la relación sujetocontexto cultural; aportando una propuesta para la sistematización de la capacidad sociocultural transformadora de los profesionales.

2. El método de la transposición sociocultural aporta un instrumento susceptible de ser empleado en los procesos de formación de los gestores culturales en contextos locales. El sistema de procedimientos del método ha posibilitado el desarrollo de experiencias de aplicación en varios entornos de actuación de estos profesionales que han sido consideradas válidas.

3. Se han desarrollado formas organizativas y experiencias que significan aportaciones al proceso de formación profesionales de la gestión cultural en los contextos locales, pues se valida la relación universidad-contexto sociocultural, como forma esencial del proceso extensionista.

4. La implementación de la estrategia pedagógica para la formación de gestores socioculturales en el contexto local de la Universidad de Granma en Cuba y la experiencia desarrollada en la Universidad Uniandes del Ecuador confirman su valía en los procesos socioformativos por su impacto tanto en la calidad como en la cantidad de gestores empoderados durante estas experiencias.

\section{Referencias}

Ausubel, David, Novak, Joseph y Hanesian, Helen. (1999). Psicología Educativa. México: Editorial Trillas.

Baczko, Bronislaw. (1971). Los imaginarios sociales. Argentina: memoria colectiva. 
Franzone, Mabel. (2007). Comprender las estructuras imaginarias de Gilbert Durand. Argentina: memoria impresa.

Freire, Paulo. (1999). Pedagogía del Oprimido. México: Editorial Siglo Veintiuno.

Fuentes González, Homero. (2009). La Formación de los profesionales en la contemporaneidad. Concepción científica holística en la Educación Superior. Santiago de Cuba: Universidad de Oriente, Centro de Estudios Educación Superior "Manuel F. Gran".

García Canclini, Néstor. (2005). Todos Tienen Cultura. Argentina: memoria impresa.

González Albear, María Isabel. (2010). Metodología Interdisciplinaria para la formación humanística en la carrera de Estudios Socioculturales, desde la disciplina Historia y Cultura. [versión digital pdf]. Recuperado de http://www.monografias.com/trabajos55/formacion-humanistica/formacionhumanistica.shtml

González Fernández-Larrea, Mercedes. (2005). Propuesta para la introducción de un modelo de gestión de la Extensión Universitaria. (Tesis para optar por el grado de Doctorado en Pedagogía), Universidad de Pinar del Rio, Pinar del Rio, Cuba.

López Calichs, Ernesto. (2011). El proceso de formación de las competencias creativas. Una vía para perfeccionar el proceso de formación profesional de los estudiantes de la carrera de licenciatura en estudios socioculturales. [versión digital pdf]. Recuperado de http://www.gestiopolis.com/recursos4/docs/ger/procomcraea.htm

Maffesoli, Michael. (2004). El Tiempo de las Tribus: el declive del individualismo en las sociedades de masa. México: siglo XXI.

Moscovichi, Sergei. (1961). La psichoanalyse son image et son Public. URSS: Paris.

Moya Padilla, Nereyda. (2009). Licenciatura en Estudios Socioculturales: Innovación curricular para la sostenibilidad. Revista Ciencias y sociedad, 34(1), 80-96. Recuperado de http://www.redalyc.org/articulo.oa?id=87014565004

Ortiz Fernández, Fernando. (1963). Contrapunteo Cubano del tabaco y el azúcar. Cuba, Consejo de Cultura.

Ponce Ruiz, Dionisio. (2011). La transposición sociocultural en la formación de los Gestores Culturales. (Tesis para optar por el grado de Doctorado en Pedagogía), Universidad de Oriente, Santiago de Cuba, Cuba. 